Canad. Math. Bull. Vol. 20 (1), 1977

\title{
AN ALGORITHM FOR THE PERMANENT OF CIRCULANT MATRICES
}

\author{
BY \\ LARRY J. CUMMINGS \\ AND \\ JENNIFER SEBERRY WALLIS
}

1. Introduction. The permanent of an $n \times n$ matrix $A=\left(a_{i j}\right)$ is the matrix function

$$
\operatorname{per} A=\sum_{\pi \in S_{n}} a_{1 \pi(1)} \cdots a_{n \pi(n)}
$$

where the summation is over all permutations in the symmetric group, $S_{n}$. An $n \times n$ matrix $A$ is a circulant if there are scalars $a_{1}, \ldots, a_{n}$ such that

$$
A=\sum_{i=1}^{n} a_{i} P^{i-1}
$$

where $P$ is the $n \times n$ permutation matrix corresponding to the cycle $(12 \cdots n)$ in $S_{n}$. In general the computation of the permanent function is quite difficult chiefly because it is not invariant under addition of a multiple of one row to another. Using the principle of "inclusion and exclusion", Ryser [6, p. 27] gave an expansion for the permanent. Also the Laplace expansion is available for the permanent $[2$, p. 20]. Neither of these methods are particularly efficient. In [4] Minc considered the permanents of matrices with entires either 0 or 1 . Minc also studied tridiagonal circulants in [5]. Metropolis, Stein, and Stein [3] have given recurrence relations for evaluating the permanents of circulant matrices (2) where the first $k$ scalars are 1 and the remaining ones are 0 . Permanents of circulant matrices were also studied by Tinsley [7].

2. The algorithm. If we consider the scalars as indeterminates over an underlying field every term of the permanent (1) of a circulant matrix (2) is a monomial in the scalars $a_{1}, \ldots, a_{n}$. Our algorithm deletes appropriate monomials from the set of all $n^{n}$ such monomials until only those appearing in the permanent remain. This is easily programmed because the monomials need only be considered one at a time and may be indexed by the $n^{n} n$-tuples chosen from $1, \ldots, n$ and ordered lexicographically. It is convenient to state the algorithm in terms of these indices.

Received by the editors Jan. 14, 1976 and, in revised form, Oct. 20, 1976. 
Algorithm. If $I=\left(i_{1}, \ldots, i_{n}\right)$ is an $n$-tuple with entries chosen from $1, \ldots, n$ then discard $I$ if

(i) $\sum_{j=1}^{n} i_{j} \neq 0(\bmod n)$,

or if

(ii) $i_{j+k} \equiv i_{j}-k(\bmod n)$ for any $k$ and $j=1, \ldots, n-1$.

Condition (ii) excludes the occurrence of terms in the permanent of (2) with the following pattern

$$
\cdots a_{i} \underbrace{* \ldots *}_{k-2 \text { entries }} a_{i+k+1} \cdots
$$

where $a_{i+n}$ is considered to be $a_{i}$ if necessary. For example, if $n=4$ condition (ii) of the algorithm discards a monomial whenever one of the following patterns occurs:

$$
\begin{aligned}
& \cdots 14 \ldots, \ldots 21 \ldots, \ldots 32 \ldots, \ldots 43 \cdots \\
& \cdot 1^{*} 3 \cdot, \cdot 2^{*} 4 \cdot, \cdot 3^{*} 1 \cdot, \cdot 4^{*} 2 \\
& 1^{* *} 2,2^{* *} 3,3^{* *} 4,4^{* *} 1
\end{aligned}
$$

Condition (i) leaves the following 4-tuples:

$\begin{array}{llllllll}1111 & 1214 & 1313 & \underline{1412} & \underline{2114} & \underline{2213} & 2312 & 2411 \\ 1124 & 1223 & \underline{1322} & \underline{1421} & \underline{2123} & 2222 & \underline{2321} & 2424 \\ \underline{1133} & \underline{1232} & \underline{1331} & \underline{1434} & \underline{2132} & 2231 & 2334 & \frac{2433}{124} \\ \underline{1142} & 1241 & 1344 & \underline{1443} & \underline{2141} & \underline{2244} & \underline{2343} & \underline{2442} \\ \underline{3113} & \underline{3212} & \underline{3311} & \underline{3414} & 4112 & \underline{4211} & \underline{4314} & 4413 \\ 3122 & \underline{3221} & \underline{3324} & 3423 & \underline{4121} & \underline{4224} & \underline{4323} & \underline{4422} \\ 3131 & \underline{3234} & 3333 & \underline{3432} & 4134 & 4233 & \underline{4332} & \underline{4431} \\ \underline{3144} & \underline{3243} & 3342 & 3441 & \underline{4143} & 4242 & \underline{4341} & 4444\end{array}$

Condition (ii) eliminates all of the above 4-tuples which are underlined.

Hence, if $n=4$ the permanent of (2) will be

$$
\sum_{i=1}^{4} a_{i}^{4}+2 a_{1}^{2} a_{3}^{2}+2 a_{2}^{2} a_{4}^{2}+4 \sum_{i=1}^{4} a_{i}^{2} a_{i+1} a_{i+3} .
$$

Let $R_{n}$ denote the set of $n$-tuples left by the algorithm. We remark that the $n$-tuples in $R_{n}$ need not be formally distinct; e.g., 1313 and 3131 are both in $\boldsymbol{R}_{\mathbf{4}}$. The number of formally distinct diagonal products in the permanent of an arbitrary circulant has been determined by Brualdi and Newman [1]. 


\section{Proofs}

THeOREM. Let $A$ be a circulant matrix (2) with scalars $a_{1}, \ldots, a_{n}$. Then

$$
\text { per } A=\sum a_{i_{1}} \cdots a_{i_{n}}
$$

where the summation is over all $\left(i_{1}, \ldots, i_{n}\right) \in R_{n}$.

Proof. We are concerned with determining conditions for which $a_{i_{1}} \cdots a_{i_{n}}$ is a term of the permanent of the $n \times n$ matrix (2). Thus, $a_{i_{k}}$ always denotes an element of the $k$ th row of (2). The $i$ th column of (2) is

$$
\left[\begin{array}{c}
a_{i} \\
a_{i-1} \\
\vdots \\
\cdot \\
a_{i-n+1}
\end{array}\right]
$$

where subscripts are taken modulo $n$. If the Laplace expansion along the first row is used to find per $A$ the entry $a_{i-k+1}$ cannot be chosen from row $k$ to appear in any monomial beginning with $a_{i}$. In any monomial of the permanent the pattern (3) cannot appear since we may expand along any row.

Therefore any $\left(i_{1}, \ldots, i_{n}\right)$ in $R_{n}$ satisfies

$$
i_{j+k} \neq i_{j}-k \text { for } k=1, \ldots, n-1 .
$$

Again, subscripts are taken modulo $n$ when necessary.

Write $i_{j+k}=i_{j}-k+x_{j k}(\bmod n)$ where $x_{j k} \neq 0,1 \leq x_{j k} \leq n-1$, and $k \neq 0$. We would like to show that $s \neq t$ implies $x_{i s} \neq x_{j t}$.

Suppose $x_{i s}=x_{j t}$. Then

Hence

$$
x_{j s}=i_{j+s}-i_{j}+s=i_{j+t}-i_{j}+t=x_{j t}
$$

but unless $s=t$

$$
i_{j+s}=i_{j+t}-(s-t) \text {, }
$$

$$
i_{j+s}=i_{j+t+(s-t)} \neq i_{j+t}-(s-t) \text {. }
$$

So assuming $x_{i s}=x_{j t}$ leads to a contradiction. Hence the contrapositive is true and $s \neq t$ implies $x_{j s} \neq x_{j t}$.

Step (i) is included in the algorithm because it is easy to implement. In fact, (ii) implies (i) as we now show:

$$
\begin{aligned}
\sum_{k=0}^{n-1} i_{j+k}=i_{j}+\sum_{k=1}^{n-1} i_{j+k} & =\left(i_{j}+\sum_{k=1}^{n-1}\left(i_{j}-k+x_{j k}\right)\right) \quad(\bmod n) \\
& =\left(n i_{j}-\sum_{k=1}^{n-1} k+\sum_{k=1}^{n-1} x_{j k}\right)(\bmod n) \\
& =\left(n i_{j}-\frac{1}{2} n(n-1)+\frac{1}{2} n(n-1)\right)(\bmod n) \\
& \equiv 0(\bmod n) .
\end{aligned}
$$


We have shown why the $n$-tuples mentioned in (i) and (ii) must be discarded. It remains to show that no more should be excluded. Condition (ii) says there are $n$ choices for $a_{i_{1}}, n-1$ choices for $a_{i_{2}}$ and in general $n-k+1$ choices for $a_{i_{k}}$. That is, condition (ii) does not eliminate exactly $n$ ! terms. But there are $n$ ! terms in the permanent so precisely the right number of monomials has been excluded.

4. Numerical results. Dr. Joan Cooper wrote a Fortran programme for our algorithm which was implemented on an ICL 1904A at the University of Newcastle, N.S.W., Australia. The following various $7 \times 7$ circulants were computed using 2.54 seconds of core time.

First row of circulant matrix $A$ row sum of

\begin{tabular}{rrrrrrrrrr}
\multicolumn{4}{c}{ circulant matrix $A$} & & & $\operatorname{per} A$ & $A=r$ & $\operatorname{per}(A / r)$ \\
\hline 3 & 1 & 1 & 0 & 1 & 0 & 0 & 4416 & 6 & 0.0157750 \\
1 & 1 & 1 & 0 & 0 & 0 & 0 & 31 & 3 & 0.0141747 \\
1 & 1 & 0 & 0 & 0 & 0 & 0 & 2 & 2 & 0.0156250 \\
1 & 1 & 1 & 1 & 1 & 1 & 1 & 5040 & 7 & 0.0061199 \\
0 & 1 & 1 & 0 & 1 & 0 & 0 & 24 & 3 & 0.0109739 \\
1 & 1 & 1 & 0 & 1 & 0 & 0 & 144 & 4 & 0.0087891 \\
1 & 1 & -1 & 0 & 0 & 0 & 0 & 1 & 1 & 1.0
\end{tabular}

We believe the algorithm is not shown to best advantage as most of the elapsed time is due to reading the 7-tuples of the example from disc.

\section{REFERENCES}

1. R. Brualdi and M. Newman, An Enumeration problem for a congruence equation, Journal of Research, (U.S.) National Bureau of Standards 74B (1970), 37-40.

2. M. Marucs and H. Minc, A Survey of Matrix Theory and Matrix Inequalities, Allyn and Bacon, Inc., Boston, 1964.

3. N. Metropolis, M. L. Stein, and P. R. Stein, Permanents of cyclic $(0,1)$ matrices, J. Combinatorial Theory 7 (1969), 291-321.

4. H. Minc, Permanents of $(0,1)$ circulants, Canad. Math. Bull. 7 (1964), 253-263.

5. H. Minc, On permanents of circulants, Pacific J. Math. 42 (1972), 477-484.

6. H. J. Ryser, Combinatorial Mathematics, MAA Carus Monograph 14, 1963.

7. M. F. Tinsley, Permanents of cyclic matrices, Pacific J. Math. 10 (1960), 1067-1082.

FACUlTy OF MATHEMATICS

UNIVERSITY OF WATERLOO

WATERLOO ONTARIO

N2L 3G1

AND

InSTITUTE OF AdVANCED STUdies

Australian National University 